\title{
Use of arrays to investigate the contribution of ATP-binding cassette transporters to drug resistance in cancer chemotherapy and prediction of chemosensitivity
}

\author{
Jian-Ting Zhang ${ }^{1}$ \\ ${ }^{1}$ Department of Pharmacology and Toxicology, Walther Oncology Center/Walther Cancer Institute and IU Cancer Center, Indiana \\ University School of Medicine, 1044 W. Walnut Street, R4-166, Indianapolis, IN 46202, USA
}

Multidrug resistance (MDR) is a major problem in cancer chemotherapy. One of the best known mechanisms of MDR is the elevated expression of ATP-binding cassette $(\mathrm{ABC})$ transporters. While some members of human $\mathrm{ABC}$ transporters have been shown to cause drug resistance with elevated expression, it is not yet known whether the over-expression of other members could also contribute to drug resistance in many model cancer cell lines and clinics. The recent development of microarrays and quantitative PCR arrays for expression profiling analysis of ABC transporters has helped address these issues. In this article, various arrays with limited or full list of $A B C$ transporter genes and their use in identifying $\mathrm{ABC}$ transporter genes in drug resistance and chemo-sensitivity prediction will be reviewed.

Keywords: genomics, MDR, drug resistance, $\mathrm{ABC}$ transporter, microarray, real time quantitative PCR

Cell Research (2007) 17:311-323. doi: 10.1038/cr.2007.15; published online 3 April 2007

\section{Introduction}

Multidrug resistance (MDR) is a major problem in cancer chemotherapy. One of the best known mechanisms of MDR is the elevated expression of ATP-binding cassette (ABC) transporters such as ABCB1 (MDR1, Pgp) [1-3], ABCC1 (MRP1) [4], and ABCG2 (BCRP, MXR, ABCP) [5] (Figure. 1). $A B C$ transporters comprise a superfamily of more than 1000 members from bacteria to human. Human alone has 49 members of the superfamily which are divided into 7 subfamilies, ABCA through ABCG (Table 1 ), and they transport a wide variety of substrates including drugs, lipids, metabolites, and ions. These human transporters are expressed in various tissues and some of them are known to function as drug efflux pumps to cause drug resistance by actively extruding multiple anticancer drugs with expenses of ATP. However, two of these human

Correspondence: Jian-Ting Zhang

Tel: +1-317-278-4503; Fax: +1-317-274-8046;

E-mail: jianzhan@iupui.edu proteins (ABCE1 and ABCF1) do not contain any known putative transmembrane domain and, therefore, may not function as transporters by themselves (see http://www. ncbi.nlm.nih.gov/books/bv.fcgi?rid=mono_001).

While some members of human $\mathrm{ABC}$ transporters have been shown to cause drug resistance with elevated expression, it is not yet known whether over-expression of other members could also contribute to drug resistance. Many model cancer cell lines selected by anticancer drugs are also available for investigating the mechanisms of drug resistance. However, dissecting which $\mathrm{ABC}$ transporter is activated in a particular case is not an easy task. Knowledge of expression profiles of $\mathrm{ABC}$ transporters and other genes involved in MDR will likely help therapeutic optimization for cancer patients in clinics. The recent development of genomic approaches such as microarrays and quantitative PCR arrays (Table 2) for expression profiling analyses has helped address these issues. In this paper, I will review recent progress in the application of these genomic tools to identify $\mathrm{ABC}$ transporter genes likely responsible for MDR in model cancer cell lines and their potential use as predictors of chemo-sensitivity. However, it should also be noted 


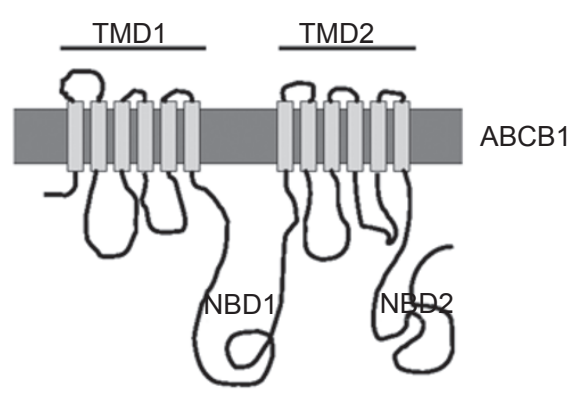

that in some cases, the findings from these profiling studies await further validation for the expression at the protein level, and the functional role of the implicated transporters in drug resistance remains to be verified.

\section{Generation of drug sensitivity predictors using high- density microarrays}

Many high density microarrays have been used to profile gene expression patterns of a large number of cell lines with known responses to multiple drugs and compounds. Correlation between the expression profiles and the drug sensitivity data of these cell lines helped generate a number of predictors for specific drugs or compounds. Some of the 49 human $\mathrm{ABC}$ transporters found in these predictors are highlighted in Table 2. However, further testing is needed to determine if these predictors are useful in clinics.

The earliest studies of drug sensitivity involved cDNA microarray analysis of the NCI-60 cell lines $[6,7]$. In these studies, a microarray containing approximately 8000 cDNA clones (Table 2) that represent unique human genes including a limited number of $A B C$ transporters such as $\mathrm{ABCA} 3, \mathrm{ABCB} 1, \mathrm{ABCB} 2, \mathrm{ABCB} 7, \mathrm{ABCC} 1, \mathrm{ABCC} 6$, and $A B C F 1$, was used. Analysis of gene expression profiles of the NCI-60 cell lines and clustering the cell lines on the basis of their responses to 1400 compounds or drugs revealed many gene-drug response relationships. It appears that the cell lines with elevated expression of ABCB1 clustered on the same branch for drug responses. This is also true for $A B C C 1$, suggesting that the expression of these $\mathrm{ABC}$ transporter genes likely contribute to the poor drug sensitivity of these cancer cell lines (Table 3). Both $\mathrm{ABCB} 1$ and $\mathrm{ABCC} 1$ are well known genes involved in resistance to multiple anticancer drugs. In addition to these $\mathrm{ABC}$ transporter genes, this study also revealed correlative gene-drug relationships for other 1376 genes. One of these genes, dihydropyrimidine dehydrogenase, was found to have a significant negative correlation between its expression and 5-FU potency in the 60 cell lines, suggesting that its over-expression may cause resistance to 5-FU.

A similar study of NCI-60 cell lines was also conducted, but instead used the Affymetrix oligonucleotide microarray Hu6800 (Table 2) containing 6817 human genes including $\mathrm{ABC}$ transporters such as $\mathrm{ABCA} 3, \mathrm{ABCA} 4, \mathrm{ABCB} 1$, $\mathrm{ABCB} 2$, and $\mathrm{ABCB} 3$ [8]. The correlation of drug sensitivity to gene expression was generated from a training set of data and this was then used to predict the sensitivity of cell lines to any particular drug using data in a testing set. Expression-based classifiers containing multiple genes for each of the 232 compounds were generated to predict drug sensitivity. Eighty-eight such classifiers performed accurately to predict drug sensitivity. Unfortunately, no 
Table 2 Hybridization and PCR-based arrays for ABC transporter expression profiling

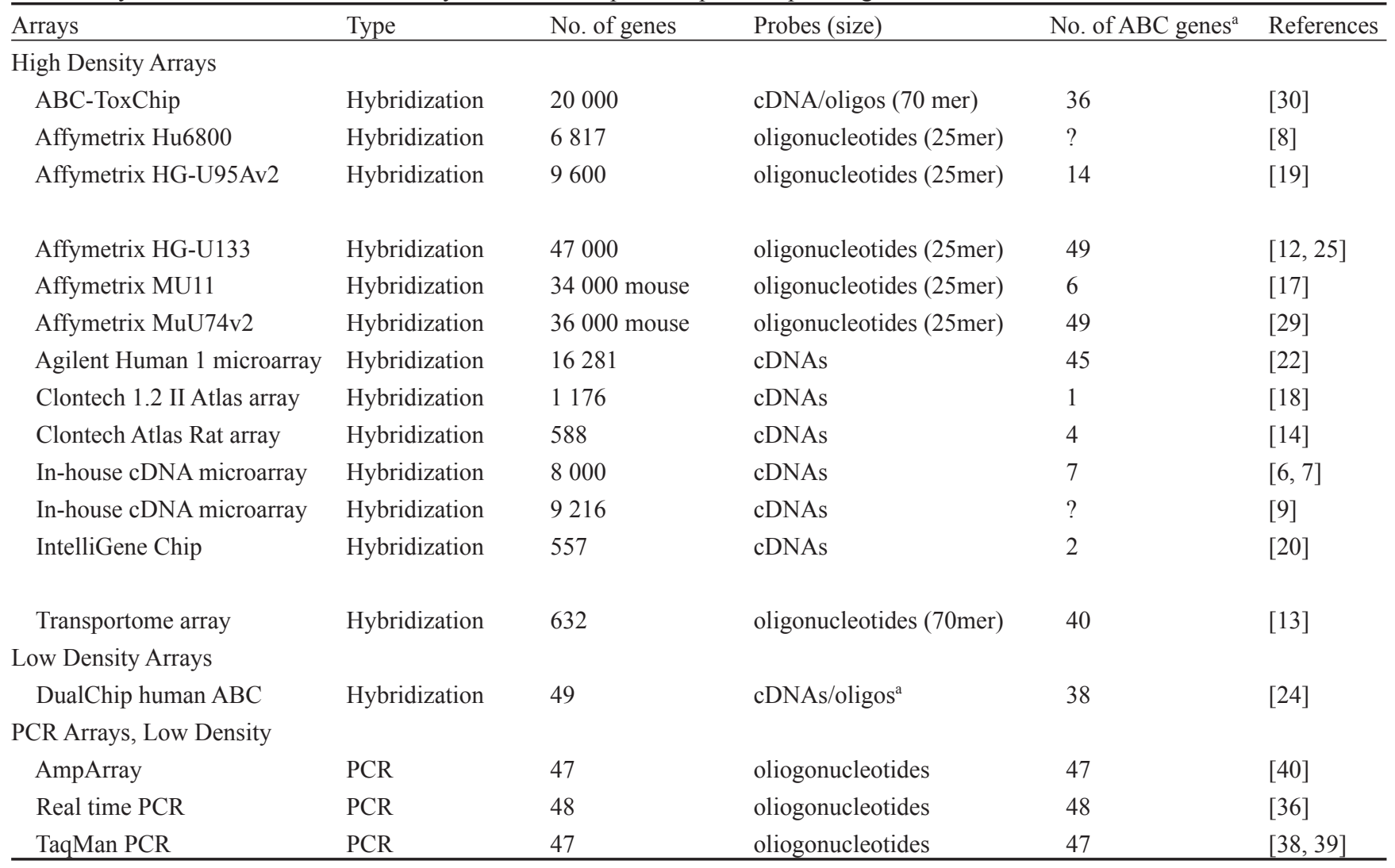

${ }^{a}$ For some arrays this information is not available in the publication nor could it be found on the internet.

Table 3 Highlight of ABC transporters identified as a predictor for poor chemo response using multiple cancer cell lines

\begin{tabular}{|c|c|c|c|}
\hline $\mathrm{ABC}$ transporters & No. of cell lines used & Drugs & References \\
\hline ABCA1 & 30 cancer cell lines & Mitoxantrone & {$[12]$} \\
\hline \multirow[t]{2}{*}{$\mathrm{ABCB} 1$} & NCI-60 cancer cell lines & Multiple & {$[6,7]^{\mathrm{b}}$} \\
\hline & NCI-60 cancer cell lines & Multiple & {$[13]^{\mathrm{c}}$} \\
\hline $\mathrm{ABCB} 5$ & NCI-60 cancer cell lines & Multiple & {$[13]^{\mathrm{c}}$} \\
\hline $\mathrm{ABCB}^{\mathrm{a}}$ & 39 cancer cell lines & Multiple & [9] \\
\hline \multirow[t]{3}{*}{$\mathrm{ABCC} 1$} & NCI-60 cancer cell lines & Multiple & {$[6,7]^{\mathrm{b}}$} \\
\hline & 30 cancer cell lines & Etopside & [12] \\
\hline & NCI-60 cancer cell lines & Multiple & {$[13]^{\mathrm{c}}$} \\
\hline $\mathrm{ABCC} 2$ & 30 cancer cell lines & $5-\mathrm{FU}$ & {$[12]$} \\
\hline $\mathrm{ABCC} 3$ & NCI-60 cancer cell lines & Multiple & {$[13]^{\mathrm{c}}$} \\
\hline ABCD3 & 30 cancer cell lines & Topotecan & {$[12]$} \\
\hline
\end{tabular}

\footnotetext{
${ }^{\text {a }}$ ABCB6 expression was reported to have positive correlation with drug sensitivity in this study

${ }^{\mathrm{b}}$ For more information see http://genome-www.stanford.edu/nci60/

${ }^{\mathrm{c}}$ For information see http://www.r-project.org

For additional information see http://dtp.nci.nih.gov/
}

particular analyses on $\mathrm{ABC}$ transporters and their roles in drug sensitivity prediction were reported in this study.

Dan et al. [9] performed a similar study using a cDNA microarray containing 9216 human genes (Table 2), but with only 39 cell lines. Of these 39 cell lines, 9 were not included in the original set of NCI-60 collection used in 
Table 4 Highlights of ABC transporters identified to have increased expression and potential contributions to drug resistance (to be continued)

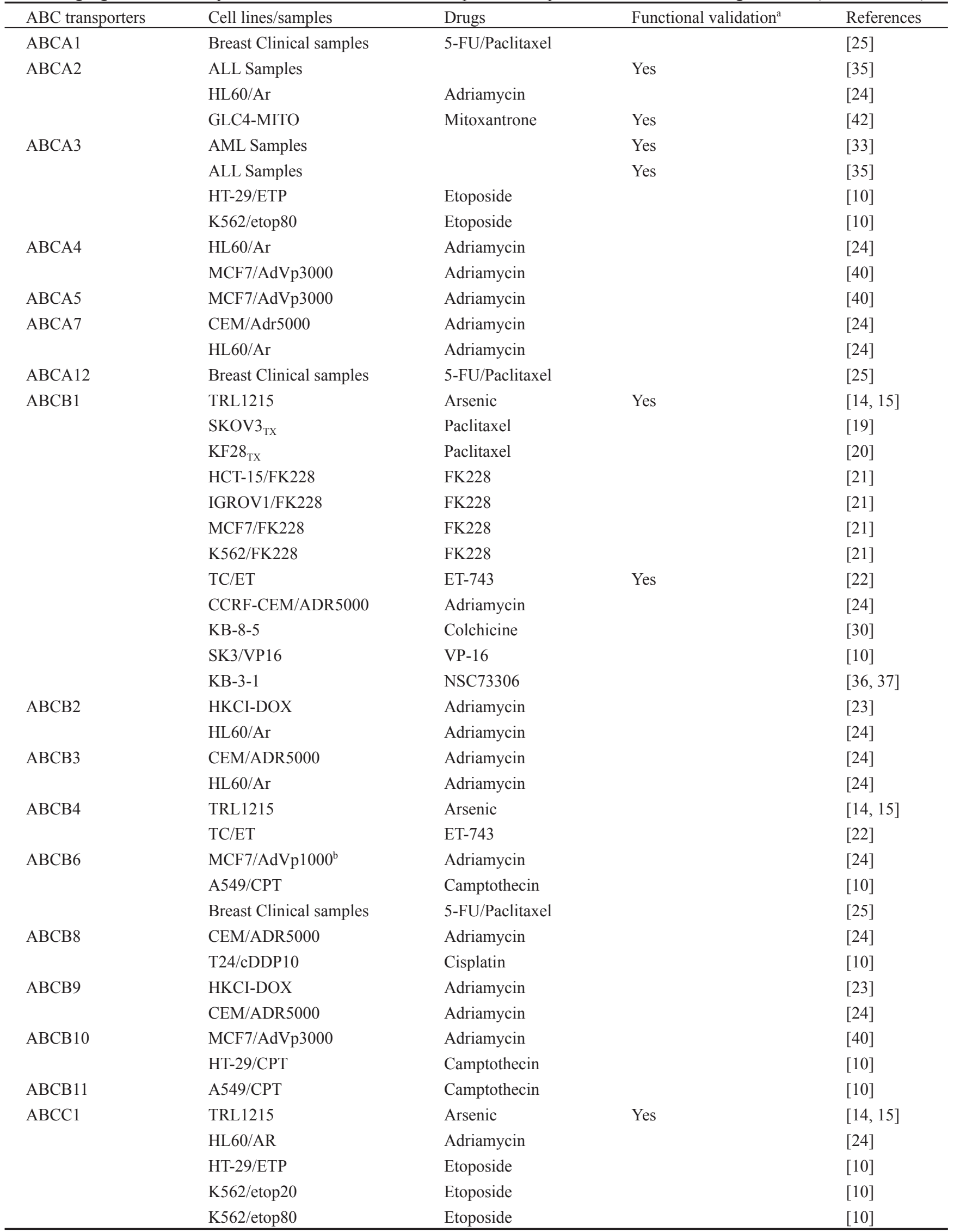


Table 4 Highlights of ABC transporters identified to have increased expression and potential contributions to drug resistance (continued)

\begin{tabular}{|c|c|c|c|c|}
\hline $\mathrm{ABC}$ transporters & Cell lines/samples & Drugs & Functional validation $^{\mathrm{a}}$ & References \\
\hline \multirow[t]{2}{*}{$\mathrm{ABCC} 2$} & RCO.1 & 9-nitro-camptothecin & & {$[30]$} \\
\hline & MCF7/AdVp3000 & Adriamycin & & [40] \\
\hline \multirow[t]{2}{*}{$\mathrm{ABCC} 3$} & MCF7/AdVp1000 & Adriamycin & & [24] \\
\hline & MCF7/AdVp3000 & Adriamycin & Yes & {$[40]$} \\
\hline \multirow[t]{2}{*}{$\mathrm{ABCC} 4$} & HL60/AR & Adriamycin & & [24] \\
\hline & KK47/cDDP10 & Cisplatin & & {$[10]$} \\
\hline \multirow[t]{3}{*}{ ABCC5 } & MCF7/AdVp1000 & Adriamycin & & [24] \\
\hline & MCF7/AdVp3000 & Adriamycin & & [40] \\
\hline & Breast Clinical samples & 5-FU/Paclitaxel & & [25] \\
\hline ABCC6 & HL60/AR & Adriamycin & & [24] \\
\hline $\mathrm{ABCC} 8$ & MCF7/AdVp3000 & Adriamycin & & [40] \\
\hline \multirow[t]{2}{*}{$\mathrm{ABCC} 9$} & SK3/VP16 & VP-16 & & [10] \\
\hline & $\mathrm{SK} 3 / \mathrm{VP}$ & VP-16 & & [10] \\
\hline ABCC11 & Breast Clinical samples & 5-FU/Paclitaxel & & [25] \\
\hline $\mathrm{ABCC} 13$ & Breast Clinical samples & 5-FU/Paclitaxel & & [25] \\
\hline $\mathrm{ABCD} 3$ & $\mathrm{SK} 3 / \mathrm{VP}$ & VP-16 & & [10] \\
\hline \multirow[t]{3}{*}{$\mathrm{ABCD} 4$} & HT-29/ADM & Adriamycin & & [10] \\
\hline & KK47/cDDP10 & Cisplatin & & {$[10]$} \\
\hline & K562/etop80 & Etoposide & & {$[10]$} \\
\hline \multirow[t]{2}{*}{ ABCE1 } & CEM/ADR5000 & Adriamycin & & [24] \\
\hline & HT-29/CPT & Camptothecin & & [10] \\
\hline \multirow[t]{2}{*}{$\mathrm{ABCF} 2$} & CEM/ADR5000 & Adriamycin & & [24] \\
\hline & T24/cDDP10 & Cisplatin & & {$[10]$} \\
\hline $\mathrm{ABCF} 3$ & MCF7/AdVp1000 & Adriamycin & & [24] \\
\hline ABCG1 & MCF7/AdVp1000 & Adriamycin & & [24] \\
\hline \multirow[t]{3}{*}{$\mathrm{ABCG} 2$} & LY/NR2 & NB-506 & Yes & [17] \\
\hline & MCF7/AdVp1000 & Adriamycin & & [24] \\
\hline & MCF7/AdVp3000 & Adriamycin & & {$[40]$} \\
\hline
\end{tabular}

${ }^{a}$ The functional validation was conducted following identification by array analyses to verify the functional role of the ABC transporter in resistance to corresponding drugs tested. Some $\mathrm{ABC}$ transporters have known roles in resistance to the corresponding drugs from prior studies, which are not counted as functional validation in this table. ${ }^{\mathrm{b}}$ This cell line was also named as MCF7/CH1000 in the original publication.

previous studies [6-8]. The drug database was also smaller with only 55 anticancer drugs. Fifty genes were found to have a significant positive (e.g., aldose reductase) or negative (e.g., LIM domain kinase 2) correlation between their expression and sensitivity to $\geq 10$ of the 55 drugs tested. Interestingly, $\mathrm{ABCB} 6$, the only $\mathrm{ABC}$ transporter gene among the 50 genes, was found to have a significant positive correlation with sensitivity to 20 anticancer drugs including Adriamycin, camptothecin, and mitoxantrone, suggesting that over-expression of $\mathrm{ABCB} 6$ may contribute to cellular sensitivity to these drugs (Table 3). ABCB6 is a mitochondrial $\mathrm{ABC}$ transporter known for its role in mitochondrial porphyrin uptake and possibly in controlling haem biosynthesis [11]. It should be noted, however, that in the study by Yasui et al., it was found that the ABCB6 gene was amplified in the camptothecin-resistant A549 cells [10]. Currently, there is no direct evidence regarding whether over-expression of ABCB6 causes resistance or sensitivity to anticancer drugs. Clearly, further studies are needed to more directly test the role of ABCB6 in drug responses.

Most recently, Gyorffy et al. [12] analyzed 30 cancer cell lines for their responses to 11 common anticancer agents and their gene expression profiles using the Affymetrix HGU133 microarray containing all 49 human ABC transporter genes (Table 2). They found that the elevated expression of 
$\mathrm{ABCA} 1$ correlates with mitoxantrone resistance, $\mathrm{ABCC} 1$ with etopside resistance, $\mathrm{ABCC} 2$ with 5 -FU resistance, and $\mathrm{ABCD} 3$ with topotecan resistance (Table 3 ). In addition, another 67 genes were found by these authors to have correlation with responses to multiple anticancer drugs. One of the notable genes is tripartite motif-containing 2 of which the expression correlates with cellular resistance to 8 different anticancer drugs.

A 70-mer oligonucleotide array containing probes for 640 transporter genes (transportome) including $40 \mathrm{ABC}$ transporters (Table 2) has also been engineered to investigate the potential contribution of membrane transporters to drug responses of cancer cell lines [13]. Using this array, many genes that encode $\mathrm{ABC}$ transporters and transporters of nucleosides, amino acids, glucose, folate, channels for calcium and water, $\mathrm{Ca}^{++}$-ATPase and $\mathrm{Na}^{+} / \mathrm{K}^{+}$-ATPase were found to significantly correlate with either drug resistance or sensitivity of the NCI-60 cell lines. Highly statistically significant negative correlations with drug sensitivity, indicative of a possible role in chemoresistance, were found for $\mathrm{ABCB} 1, \mathrm{ABCB} 5, \mathrm{ABCC}$, and $\mathrm{ABCC} 3$ (Table 3). The differential expression of these genes, except for $\mathrm{ABCC} 1$, was also confirmed using real time RT-PCR. While ABCB1, $A B C C 1$, and $A B C C 3$ were known to cause drug resistance, the expression of $\mathrm{ABCB} 5$, which was not previously known to affect drug responses, was found to negatively correlate with camptothecin and 1,2,4,5-Tetramethylbenzene sensitivity. The follow-up study using siRNA to down-regulate ABCB5 expression in melanoma SK-MEL-28 cells caused sensitization to several anticancer drugs including camptothecin, 10-OH camptothecin, and 5-FU. These findings suggest that $\mathrm{ABCB} 5$ over-expression likely also causes MDR and may serve as a predictor along with $\mathrm{ABCB} 1$, $\mathrm{ABCC} 1$, and $\mathrm{ABCC} 3$ for poor drug sensitivity.

In addition to $\mathrm{ABC}$ transporters, other transporters have also been found to have significant correlations with drug responses by using the transportome array. While some of them such as the folate carrier are known to affect sensitivity to drugs such as methotrexate, others such as amino acid transporters have not been implicated in such a process. However, the sufficient structural similarity between amino acids and their analogue drugs may warrant the involvement of these transporters in drug responses of these cancer cells. This speculation awaits verification from future studies.

\section{Identification of $\mathrm{ABC}$ transporter genes in drug-se- lected cell lines using high density microarrays}

Comparative gene expression profiling has been conducted by using the Clontech Atlas Rat cDNA array (Table 2) containing 588 genes, including $4 \mathrm{ABC}$ transporters (ABCB1, $\mathrm{ABCB} 4, \mathrm{ABCC} 1$, and $\mathrm{ABCC}$ ), to investigate genes potentially responsible for arsenic tolerance in chronically exposed rat liver epithelial cell line TRL1215 [14]. Compared with the parental arsenic sensitive TRL1215 cells, the arsenic resistant TRL1215 has $\sim 80$ genes with altered expressions. Of these genes, $\mathrm{ABCB} 1, \mathrm{ABCB} 4$, and $\mathrm{ABCC} 1$ were among the genes with increased expression (Table 4). In a follow-up study, Liu et al. verified the altered expression of these three $\mathrm{ABC}$ transporters by using RT-PCR and western blot analyses [15]. They further demonstrated that inhibition of ABCB1 and $\mathrm{ABCC} 1$ function using their respective inhibitors could significantly increase arsenic sensitivity of the arsenic tolerant TRL1215 cell line, suggesting that over-expression of $\mathrm{ABCB} 1$ and $\mathrm{ABCC} 1$ may contribute to arsenic tolerance. However, it is not yet known if arsenic or its metabolites are directly effluxed by these ABC transporters. Furthermore, because the rat array contains only a limited number of $A B C$ transporter genes, its usefulness is likely limited as it can not identify the potential roles of other $\mathrm{ABC}$ transporters in arsenic tolerance.

The anticancer drug NB-506, a Topo I inhibitor, has been shown not to be a substrate of the known drug transporter $\mathrm{ABCB} 1$ or $\mathrm{ABCC} 1$ [16], although NB-506 resistant cell lines showed decreased intracellular accumulation of NB-506 [17]. To discover genes that could be involved in NB-506 resistance and elimination, Komatani et al. performed comparative genomic analyses using Affymetrix arrays (Mu11K and Mu19K, Table 2) containing over 34 000 mouse genes [17]. They found that ABCG2, in addition to 11 other genes, had the most prominent elevation in expression in NB-506 resistant mouse fibroblast cell line LY/NR2 compared with the parental cell line LY (Table 4). The role of ABCG2 in cellular resistance to NB-506 was validated subsequently by ectopic over-expression of ABCG2 in the lung cancer cell line PC-13 [17].

In a similar study to discover resistance mechanisms of Adriamycin-selected acute myeloid leukemia cell line K562/A02 using the Clontech Human 1.2 II Atlas array (Table 2) containing only $1 \mathrm{ABC}$ transporter gene (ABCD1), Tan et al. found 12 differentially expressed genes between K562/A02 and its parental sensitive line K562 [18]. Because of the limited ABC transporter genes on the array, no $\mathrm{ABC}$ transporter gene would have been found here despite the fact that $\mathrm{ABCB} 1$ is known to be strongly over-expressed in the K562/A02 cell line. Thus, the usefulness of this array for $\mathrm{ABC}$ transporter analysis is very limited.

A regular Affymetrix HG-U95Av2 array (Table 2) containing 9600 known human genes including $14 \mathrm{ABC}$ transporters was used to identify molecular signatures that are possibly responsible for paclitaxel resistance in 3 stepwise 
selected SKOV3 ovarian cancer cell lines [19]. Of the 14 $\mathrm{ABC}$ transporter genes collected on this array, only $\mathrm{ABCB} 1$ was found to have increased expression correlative with the sequential increase in drug resistance of the 3 stepwise selected cell lines. Interestingly, the expression of ABCC 3 and $\mathrm{ABCC} 4$ was decreased in the drug resistant cell lines. In a similar study of ovarian cancer cell line $\mathrm{KF} 28_{\mathrm{TX}}$ resistant to paclitaxel using an IntelliGene microarray (Table 2) containing 557 human cancer-related cDNAs including $\mathrm{ABCB} 1$ and $\mathrm{ABCC} 3, \mathrm{ABCB} 1$ was also found to be overexpressed and likely contribute to paclitaxel resistance [20]. Similar to the Clontech Rat cDNA array (see above), the usefulness of these arrays in $\mathrm{ABC}$ transporter profiling, however, is also limited.

In the study using Affymetrix HG-U95Av2 array [19], some other genes in addition to $\mathrm{ABC}$ transporters, were also found to have increased expression either in the early cell line with low paclitaxel resistance level or in the later cell line with intermediate or high level of resistance; and they were clustered into 3 categories based on their emerged increase in the 3 stepwise selected cell lines. The early respondent genes are primarily inflammatory response genes such as IL-8. The intermediate genes include carrier and primary active transporter genes. The late respondent genes include tumor and cell surface antigens and signal transducers. Still many other genes were also found to have altered expression in the cell line either with low or higher level of resistance. However, the expression of these genes did not follow the evolution trend with increased expression correlating with the increased drug resistance in the 3 stepwise selected cell lines. Therefore, their contributions to paclitaxel resistance in ovarian cancer cells may be limited.

Using the same transportome array developed by Huang et al. [13] (see above), Xiao et al. analyzed the underlying mechanism of acquired resistance of cancer cell lines HCT15, IGROV1, MCF7, and K562 to the histone deacetylase inhibitor FK228 [21]. These authors found that ABCB1 expression is dramatically up-regulated in all four FK228resistant derivative cell lines (Table 4). It was also found that $\mathrm{ABCB} 1$ expression was induced by FK228 likely via alterations in histone acetylation at the $\mathrm{ABCB} 1$ promoter. However, ABCB1-mediated efflux of FK228 and its direct involvement in FK228 resistance have not yet been established.

ET-743, a new anticancer agent derived from marine products, is currently under clinical trial; and variation in responses to this drug has been observed, suggesting that clinical resistance to ET-743 may develop. Using the Human 1 cDNA microarray containing 16,281 cDNA probes with $45 \mathrm{ABC}$ transporter genes from Agilent Technologies (Table 2), Manara et al. profiled the differential gene expression between two ET-743 resistant Ewing's sarcoma cell lines, TC/ET $6 \mathrm{nM}$ and TC/ET $12 \mathrm{nM}$, and their parental sensitive cell line TC-71 [22]. The expressions of sixty-five genes were found commonly changed in the two resistant sublines compared with the sensitive cells. Among these genes, ABCB1 and ABCB4 were upregulated most prominently in the resistant cells (Table 4). Using comparative genomic hybridization, $\mathrm{ABCB} 1$, but not $\mathrm{ABCB} 4$, was also found to have been amplified in the resistant cells. Since ABCB1 is the gene with most prominent increases in expression, it is likely very important for ET-743 resistance. Indeed, inhibition of ABCB1 activity by PSC-833 dramatically reduced ET- 743 resistance level [22]. However, the potential contribution of the remaining 64 genes including ABCB4 to ET-743 resistance has not yet been rigorously examined.

The mechanism of Adriamycin resistance in five pairs of hepatocarcinoma cell lines has also been examined using a 19 K EST microarray [23] (Table 2). The expression of over 60 genes including $\mathrm{ABCB} 2$ and $\mathrm{ABCB} 9$ was found to have commonly increased in the 5 resistant sublines compared with their sensitive parental hepatocellular carcinoma cells. While ABCB2 participates in peptide transport in ER for antigen presentation, the function of $\mathrm{ABCB} 9$ was not yet known. Neither of these two genes has been shown previously to cause drug resistance. However, in the profiling study by Gillet et al. using their low density DualChip human $\mathrm{ABC}$ microarray (see below), $\mathrm{ABCB} 2$ and $\mathrm{ABCB} 9$ were also found to be over-expressed in Adriamycin-resistant HL-60 and CCRF-CEM cell lines, respectively [24] (see below). It is currently unknown if the over-expression of $\mathrm{ABCB} 2$ and $\mathrm{ABCB} 9$ causes Adriamycin resistance.

The high density Affymetrix arrays have also been used for focused analysis of $\mathrm{ABC}$ transporter gene expression despite the fact that they contain many more genes. One of these studies was performed using Affymetrix HG-U133 Plus 2.0 GeneChip which contains all 49 human ABC transporter genes (Table 2). ABC transporter expression profiles were generated using this array from breast cancer patients who received neoadjuvant chemotherapy including 5-FU and paclitaxel [25]. Comparison between patients who had complete response and those who had residual disease revealed that the expression of $A B C A 1, A B C A 12$, $\mathrm{ABCB} 6, \mathrm{ABCC} 5, \mathrm{ABCC} 11, \mathrm{ABCC} 13$ was elevated in the residual disease group, suggesting that their expression may contribute to the incomplete response of these patients to neoadjuvant therapy. Of these transporters, ABCC5 was found to be the elevated gene with the highest significance and highest expression level (Table 4). ABCC5 has been shown previously to cause resistance to several drugs including 5-FU $[26,27]$. Thus, its high expression level in breast cancers may contribute to clinical resistance to drugs such as 5-FU. Gillet et al. also performed an analysis of 
16 breast cancer samples using their low density DualChip human $\mathrm{ABC}$ microarray (see below) and found $23 \mathrm{ABC}$ transporters including $\mathrm{ABCC} 1$ and $\mathrm{ABCC} 5$ were expressed in the majority ( $\geq 10$ of 16 ) of the breast cancer samples tested [28]. Unlike the study by Park et al. [25], however, the Gillet study [28] did not have the correlation analysis between patient response to chemotherapy and their $\mathrm{ABC}$ transporter expression level. Thus, the implication of $A B C$ transporter expression in this study is limited.

The Affymetrix MuU74v2 GeneChip with 33000 mouse genetic elements contains annotated oligonucleotide probes for 43 of the 51 mouse $\mathrm{ABC}$ transporter genes (Table 2). Using this array, Mutch et al. [29] conducted a focused analysis of $\mathrm{ABC}$ transporter expression profiles along the intestinal tract of mice. While most $\mathrm{ABC}$ transporters do not show differential expressions in the duodenum, jejunum, and colon, $8 \mathrm{ABC}$ transporter genes $(\mathrm{ABCB} 2, \mathrm{ABCB} 3$, $\mathrm{ABCB} 9, \mathrm{ABCC} 3, \mathrm{ABCC} 6, \mathrm{ABCD} 1, \mathrm{ABCG} 5$, and $\mathrm{ABCG} 8$ ) show significant alterations in expression along the intestinal tract. The alterations in expression of these 8 genes have also been validated using real-time PCR. These findings suggest that the Affymetrix MuU74v2 GeneChip may also be used for a focused analysis to investigate differential $\mathrm{ABC}$ transporter gene expression in drug resistant mouse cell lines compared with their parental sensitive lines.

Gottesman's laboratory has developed a high density ABC-ToxChip for microarray analysis of toxicological response genes [30]. On this microarray, in addition to 36 human $\mathrm{ABC}$ transporter genes, a comprehensive set of detoxifying genes were also included. By comparing the KB-3-1 cell line with its corresponding resistant derivative cell line selected using colchicine (KB-8-5), they showed that $\mathrm{ABCB} 1$ had dramatic over-expression in the KB-8-5 cell line (Table 4), the known mechanism of resistance in this cell, thus validating the usefulness of the array. Using this array to analyze DU-145 cell line and its corresponding resistant derivative cell line (RCO.1) selected using 9-nitro-camptothecin revealed that RCO.1 over-expresses ABCC2 (Table 4), suggesting that ABCC2 may contribute to 9-nitro-camptothecin resistance. However, the notion that over-expression of $\mathrm{ABCC} 2$ causes 9-nitro-camptothecin resistance has not yet been validated. Furthermore, RCO. 1 also over-expresses many other genes of the 20000 genes tested such as the EGF-like repeats and discoidin I-like domain protein (U70312), which may also contribute to 9-nitro-camptothecin resistance.

\section{Identification of $\mathrm{ABC}$ transporter genes in drug-se- lected cell lines using low density microarrays}

Use of high-density arrays to profile only $\mathrm{ABC}$ transporter expression is apparently not cost-effective. Recently, low density arrays containing only $\mathrm{ABC}$ transporter genes have been developed which can be used more cost-effectively for focused studies of $\mathrm{ABC}$ transporters in drug resistant versus sensitive cells (Table 2). The first of such arrays contains 38 human ABC transporter genes (DualChip human $\mathrm{ABC}$ low-density microarray) and it has been validated using three MDR cell lines, CEM/ADR5000, HL60/AR, and MCF7/AdVp1000 in comparison with their respective parental CEM, HL60, and MCF7 cells [24]. Using this array it was found that CEM/ADR5000, HL60/AR, and MCF7/AdVp1000 over-expressed ABCB1, ABCC1, and ABCG2, respectively, as previously demonstrated $[5,31$, 32], thus validating the usefulness of this low density array. In this study, several other $\mathrm{ABC}$ transporters were also found to be over-expressed in the drug resistant cell lines (Table 4), suggesting that they may also contribute to the resistance selected in these cells. However, none of these additional $\mathrm{ABC}$ transporter genes was validated for their functional role in drug resistance.

The same array was also used to profile ABC transporter expression and its correlation to clinical responses of 42 childhood acute myeloid leukemia (AML) patients [33]. It was found that, of all 38 human $\mathrm{ABC}$ transporters examined, only ABCA3 had significant correlation of over-expression to poor response (Table 4), suggesting that ABCA3 may contribute to clinical drug resistance in AML. Knocking down $\mathrm{ABCA} 3$ expression in the osteosarcoma cell line 143B with siRNA caused a statistically significant increase in sensitivity to Adriamycin. Nevertheless, this increase in sensitivity is very small $(<20 \%)$ [33]. Because ABCA3 is normally located at intracellular membranes [34], it seems unlikely to mediate drug resistance by directly effluxing drugs as do the traditional $\mathrm{ABC}$ drug transporters such as ABCB1 and ABCC1.

In another study, the same research group analyzed 21 childhood $\mathrm{T}$ cell acute lymphoblastic leukemia (ALL) samples using the same DualChip human ABC low-density microarray and found consistent over-expression of $\mathrm{ABCA} 2$ and $\mathrm{ABCA} 3$, similar to the findings in childhood myeloid leukemia [35]. However, correlation analysis between $\mathrm{ABC}$ transporter expression and the clinical outcome did not generate any statistically significant differences in the ALL. Although ABCA3 knock-down in the osteosarcoma cell line 143B by siRNA appeared to generate a significant increase in cellular sensitivity to Adriamycin $[33,35]$, it did not result in any survival effect in response to vinblastine and methotrexate. Knock-down of ABCA2 alone in 143B cells did not appear to have any effect on survival in response to Adriamycin, vinblastine, or methotrexate. However, the combined knock down of ABCA2 and ABCA 3 resulted in a significant increase in cell death in response to all three anticancer drugs [35], 


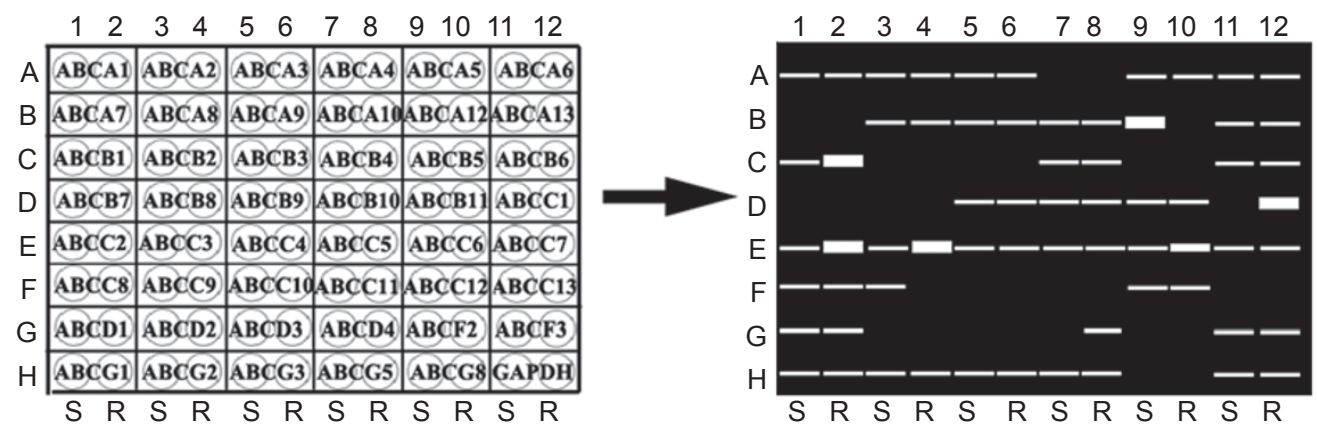

Figure 2 Schematic presentation of AmpArray template and its application. The template of AmpArray for 47 ABC transporters in a 96-well plate format is shown on the left. The right image is a schematic presentation of a theoretical agrose gel image demonstrating the comparative level of each $\mathrm{ABC}$ transporter between sensitive $(\mathrm{S})$ and resistant $(\mathrm{R})$ cells.

suggesting a potential compensatory effect between the two $\mathrm{ABC}$ transporters.

\section{Quantitative PCR arrays to identify ABC transporter genes}

The same 60 NCI cell lines discussed above were also examined for the correlation of their drug sensitivities with the expression of 48 human $\mathrm{ABC}$ transporters using a focused real-time RT-PCR approach [36] (Table 2). Correlation between the expression of $\mathrm{ABC}$ transporter genes and resistance to candidate drugs was analyzed. While there were interesting positive correlations between $\mathrm{ABC}$ transporter expression and resistance to drugs, along with the discovery of some previously unknown substrates for certain $\mathrm{ABC}$ transporters such as $\mathrm{ABCB} 1$, negative correlations between $\mathrm{ABC}$ transporter expression and drug resistance were also observed. One of the novel compounds that have positive correlations in resistance with $\mathrm{ABCB} 1$ expression is NSC363997. MTT assay using KB-3-1 and the drug-selected $\mathrm{KB}-\mathrm{V} 1$ cells, which over-express ABCB1, showed that NSC363997 is likely an ABCB1 substrate [36]. In contrast, NSC73306 is an example for compounds that have negative correlations in resistance with ABCB1 expression. Validation studies showed that cells over-expressing $\mathrm{ABCB} 1$ are less resistant to NSC73306 [36]. More recently, Ludwig et al. followed up on the observation of NSC73306 and found that NSC73306 toxicity is actually mediated by $\mathrm{ABCB} 1$ over-expression [37]. Inhibiting the function of $A B C B 1$ using inhibitors or decreasing its expression using siRNA both decreased cellular sensitivity to NSC73306. Interestingly, long term selection of several cancer cell lines with NSC73306 also resulted in NSC73306-resistant derivative cells, which apparently have decreased expression of ABCB1. These findings are interesting because $\mathrm{ABCB} 1$ over-expression has previously been recognized only to cause resistance but not sensitivity to drugs. Nevertheless, the mechanism of ABC-transporter-mediated cytotoxicity is currently unknown and further studies are needed to interpret this phenomenon and investigate the mechanism of action.

The TaqMan real-time RT-PCR approach involving 47 $\mathrm{ABC}$ transporters has been developed by another group [38] to profile $\mathrm{ABC}$ transporter expression in human tissues. The expression profiles of $\mathrm{ABC}$ transporters in human tissues appear to be consistent with their known ubiquitous distribution. However, some ABC transporters show restricted expression patterns such as ABCG5 and ABCG8 in liver and intestine. The utility of this approach for analyzing drug resistant cells has yet to be tested.

While the above real-time PCR approach represents a focused assay on $\mathrm{ABC}$ transporters, performing this assay routinely on all $\mathrm{ABC}$ transporter genes is time-consuming. Recognizing this problem, Langmann et al. [39] recently improved their TagMan real-time RT-PCR approach by developing a low-density array of $47 \mathrm{ABC}$ transporters based on an Applied Biosystems 7900HT Micro Fluidic Card, which allows simultaneous PCR analysis of 47 ABC transporters (Table 2). Although this array has not been tested for analyzing drug resistant cells, it was used to analyze the expression profiles of $\mathrm{ABC}$ transporters in primary human monocytes, differentiated macrophages, and macrophages treated with LXR and RXR agonist T0901317 and retinoic acid. It was found that $30 \mathrm{ABC}$ transporters were expressed in human primary monocytes. Following differentiation into macrophages, the number of expressed $\mathrm{ABC}$ transporters decreased to 26. Although the expression level of most of these $\mathrm{ABC}$ transporters is lower than that in monocytes, $\mathrm{ABCB} 1, \mathrm{ABCB} 11$, and $\mathrm{ABCG} 2$ are the new genes detected while the expression of $\mathrm{ABCB} 9$ and $\mathrm{ABCC} 5$ is increased 
in macrophages. Treatment of macrophages with LXR and RXR agonist T0901317 and retinoic acid further decreased the number of expressed $\mathrm{ABC}$ transporter genes to 12 . It may be too premature to conclude that $\mathrm{ABCB} 1, \mathrm{ABCB}$, $\mathrm{ABCB} 11, \mathrm{ABCC} 5$, and $\mathrm{ABCG} 2$ expression plays important roles in differentiation. However, future detailed studies should help answer the question regarding the relationship between these $\mathrm{ABC}$ transporters and differentiation.

Another simpler and inexpensive AmpArray approach (Figure 2, Table 2) has also been designed recently and tested for investigating the expression profiles of $47 \mathrm{ABC}$ transporters using RT-PCR on a 96-well plate [40]. This comparative assay is quick, convenient, and economical, as $47 \mathrm{ABC}$ transporters are assayed at the same time on a regular thermal cycler that houses a 96-well plate format. This approach was tested using parental cell line MCF7 and its drug resistant derivative subline MCF7/AdVp3000. It was found that $\mathrm{ABCG} 2$ expression was dramatically increased in the resistant MCF7/AdVp3000 cells, which are consistent with previous findings that increased ABCG2 expression is a major cause of resistance in MCF7/AdVp3000 cells [5]. Interestingly, several other ABC transporters were also found to show altered expression levels. Particularly, the expression of $\mathrm{ABCA} 4$ and $\mathrm{ABCC} 3$ was drastically increased in the drug resistant subline and then reversed completely along with ABCG2 in the revertant MCF7/AdVpRev cells as determined by real-time PCR. The altered expression and possible contribution of $\mathrm{ABCC} 3$ to Adriamycin and mitoxantrone resistance were also validated by using western blot and SRB assays, respectively. However, the altered expression of ABCA4 could not be confirmed at the protein level. These findings suggest that more than one $\mathrm{ABC}$ transporters could be up-regulated to cause resistance in drug selected cell lines and that the altered expression at the mRNA level as determined by arrays or PCR does not necessarily correlate with the level of the corresponding protein. Compared to the real-time PCR low-density arrays, which require the use of specialized instrumentation and reagents, the AmpArray analysis is less expensive and simpler to perform. However, AmpArray suffers from its semi quantitative nature.

\section{Comparative genomic approaches to investigate $\mathrm{ABC}$ transporter gene amplifications and their contribu- tion to drug resistance}

In addition to microarrays and real time quantitative PCRs to investigate the potential involvement of $A B C$ transporters in drug resistance, other approaches have recently been developed. One of these approaches is subtractive comparative genomic hybridization of metaphase DNA on slides [41]. By using this approach to profile ABC transporter gene amplifications in 23 drug resistant cancer cell lines, Yasui et al. recently found that $\mathrm{ABCA} 3, \mathrm{ABCB} 1$, ABCB6, ABCB8, ABCB10, ABCB11, ABCC1, ABCC4, $\mathrm{ABCC} 9, \mathrm{ABCD} 3, \mathrm{ABCD} 4, \mathrm{ABCE} 1$, and $\mathrm{ABCF} 2$ were amplified in various drug resistant cancer cell lines relative to their parental sensitive ones (Table 4). The amplified genes of $\mathrm{ABCA} 3, \mathrm{ABCC} 1$, and $\mathrm{ABCC} 9$ were also shown to have increased expression in the drug resistant cells at the RNA level using northern blot or RT-PCR analyses. However, it is currently unknown if amplifications of other $\mathrm{ABC}$ transporter genes also cause their over-expression in the drug resistant cells. Such a potential problem was better addressed in another similar study by Boonstra et al. who also used the similar subtractive comparative genomic hybridization to analyze a mitoxantrone resistant small cell lung cancer cell line GLC4-MITO [42]. Of the 7 amplified $\mathrm{ABC}$ transporter genes (ABCA2, ABCB2, $\mathrm{ABCB} 3, \mathrm{ABCB} 6, \mathrm{ABCC} 4, \mathrm{ABCC} 10$ and $\mathrm{ABCF} 1)$ detected using comparative genomic hybridization, only $\mathrm{ABCA} 2$ was found to show corresponding over-expression at RNA and protein levels, and its over-expression likely causes mitoxantrone resistance (Table 4). This observation suggests that caution should be taken when the comparative genomic hybridization approach is used and validations using other approaches to determine the expression level should be performed.

\section{Concluding remarks and perspectives}

Both high and low density microarrays and quantitative PCR approaches with limited or full list of ABC transporter genes have been developed and tested. These arrays clearly can be used for a variety of studies to profile $A B C$ transporter gene expression in drug resistant cancer cell lines or clinical tissues, which could not have been accomplished in the past. Predictors for drug sensitivity can also be built from the results of $\mathrm{ABC}$ transporter expression profiling which may assist the design of individualized therapies.

It is clear from the studies reviewed here that any given drug resistant cancer cell line likely has multiple ABC transporters selected for contribution to resistance (Table 4). Although the most frequently selected $\mathrm{ABC}$ transporters in various drug resistant cell lines appeared to be $\mathrm{ABCB} 1$ and $\mathrm{ABCC} 1$, consistent with their known roles in MDR, new roles in drug resistance previously unknown for some $\mathrm{ABC}$ transporters were also found using these profiling analyses. For example, $\mathrm{ABCC} 3$ and $\mathrm{ABCC} 5$ were both found upregulated in the Adriamycin-selected MCF7/AdVp1000 [24] and MCF7/AdVp3000 [40] cell lines using two different arrays, strongly suggesting that these transporters may contribute to Adriamycin resistance. Although these transporters may be selected by verapamil that was present 
during the selection of these cell lines, the role of $\mathrm{ABCC} 3$ in Adriamycin resistance has been validated functionally by the siRNA knock down strategy [40]. Clearly, further studies are still needed to more definitively demonstrate that the over-expression of these transporters causes Adriamycin resistance.

The findings that drug resistant cancer cells may overexpress multiple $\mathrm{ABC}$ transporters simultaneously impose a challenge for successful clinical cancer chemotherapy. Sensitization of the drug resistance phenotype to optimize cancer chemotherapy may require considerations of inhibiting multiple drug efflux $\mathrm{ABC}$ transporters. It is noteworthy that in some studies members of $\mathrm{ABC}$ transporters were found to have decreased expression in drug resistant cells. It is currently unknown whether the decreased expression of these $\mathrm{ABC}$ transporters contributes to drug resistance. In other studies, it has been found that the over-expression of $\mathrm{ABC}$ transporter mediates cytotoxicity of some compounds. Again, the mechanism of action is unknown. Clearly, more studies are needed to investigate if decreased expression of some $\mathrm{ABC}$ transporters causes drug resistance and how certain $\mathrm{ABC}$ transporter over-expression mediates cytotoxicity of some drugs.

Based on the studies reviewed here, it is clear that while mass information could be produced from the array profiling studies for potential drug resistance contributors, most of the studies are correlative and lack functional validation of the implicated genes that may be responsible for the drug resistance/sensitivity observed. Of course, simultaneous analyses of cell lines with intermediate drug resistance generated during stepwise selections and revertant cell lines that have lost resistance as demonstrated in the studies by Lamendola et al. [19] and Liu et al. [40] would help identify genes that likely contribute to the resistance of the cells. Further functional validation of these genes in drug resistance (Table 3) using siRNA-mediated silencing, ectopic over-expression, and known inhibitors would be very fruitful and could help verify the new drug resistance functions of these $\mathrm{ABC}$ transporters.

Another problem associated with $\mathrm{ABC}$ transporter gene profiling using microarrays or quantitative PCRs is that these approaches only detect the changes in expression at the mRNA level. The protein level of the corresponding transporter may or may not be changed. For example, the expression of ABCA4 in the AmpArray study by Liu et al. [40] was increased at the mRNA level in the drug resistant MCF7/AdVp3000 cells, as detected by the array profiling and confirmed by real time RT-PCR. However, the change of ABCA4 protein was not detected using several antibodies. Rather than validating the gene expression using real time PCR, as shown in most of the studies discussed above, western blot analyses would be preferred if antibodies are available. Furthermore, immunohistochemistry using tissue microarrays has been developed to examine the expression of $\mathrm{ABC}$ transporters, which would also facilitate the study using clinical human tissue samples and eliminate the potential problems from detection of mRNAs as mentioned above [43, 44]. Proteomic approaches have also been developed to investigate drug resistance mechanisms and may help solve the aforementioned problem of gene profiling studies. However, the proteomic approach can be problematic and limited in separating and identifying polytopic membrane proteins including $\mathrm{ABC}$ transporters (Zhang and Liu, manuscript submitted).

Because $\mathrm{ABC}$ transporters share high homology, there is a potential problem of cross hybridization associated with microarrays, although the oligonucleotide arrays contain multiple probes targeting different regions of each target. As suggested by Lee et al. [45], performing similar studies using both cDNA and oligonucleotide microarrays, which are subjected to different artifactual influences during hybridization, may help minimize this potential problem. For quantitative PCR approaches, the use of certain reference genes such as GAPDH can also be problematic. It has been found that GAPDH expression was increased in the Adriamycin-resistant MCF7/Adr cells [46, 47]. In a metabolic marker-focused proteomic profiling analysis of 101 breast cancer tissues, Isidoro et al. [48] also found that the expression of GAPDH was significantly increased in patients with poor survival. These studies suggest that GAPDH may not be an ideal internal reference gene for the profiling studies using PCR. Most recently, Calcagno showed that plasma membrane calcium-ATPase 4 (PMCA4) is a better reference gene for real time PCR quantification of $\mathrm{ABC}$ transporter genes [49]. PMCA4 is a ubiquitously expressed house keeper plasma membrane protein and its expression in several cancer cell lines appeared to be unaffected by treatment with various agents including anticancer drugs.

Some arrays are limited in the number of ABC transporter genes (Table 1), and thus, their usefulness in profiling $\mathrm{ABC}$ transporter expression is likely also limited. The past studies using these arrays would likely have missed some $\mathrm{ABC}$ transporter genes that have bona fide roles in drug resistance. Furthermore, using the low density arrays and quantitative PCRs also likely would miss information about other potential mechanisms of drug resistance when profiling drug resistant and sensitive cancer cells, although the low density arrays are more cost effective. Taken together, it is clear that comprehensiveness, cost effectiveness, simplicity of operation, and fidelity should all be considered when choosing any of these comparative genomic profiling approaches. At least two independent approaches are recommended to validate the findings. 
Furthermore, it is known that $\mathrm{ABC}$ transporter polymorphism such as that in ABCG2 [50] could affect substrate specificity and, thus, resistance to certain anticancer drugs. These polymorphisms of $\mathrm{ABC}$ transporters have not yet been considered in the $\mathrm{ABC}$ transporter arrays developed thus far. Future studies to develop $\mathrm{ABC}$ transporter arrays with consideration of polymorphisms would be important for studies of MDR and drug responses.

\section{Acknowledgment}

This work was supported in part by the National Institutes of Health Grants CA94961 and CA120221. Editorial proof reading by Jeff Russ is appreciated.

\section{References}

1 Gerlach JH, Endicott JA, Juranka PF, et al. Homology between P-glycoprotein and a bacterial haemolysin transport protein suggests a model for multidrug resistance. Nature 1986; 324: 485-489.

2 Chen CJ, Chin JE, Ueda K, et al. Internal duplication and homology with bacterial transport proteins in the $\mathrm{mdr} 1$ (P-glycoprotein) gene from multidrug-resistant human cells. Cell 1986; 47:381389.

3 Gros P, Croop J, Housman D. Mammalian multidrug resistance gene: complete cDNA sequence indicates strong homology to bacterial transport proteins. Cell 1986; 47:371-380.

4 Cole SP, Bhardwaj G, Gerlach JH, et al. Overexpression of a transporter gene in a multidrug-resistant human lung cancer cell line [see comments]. Science 1992; 258:1650-1654.

5 Doyle LA, Yang W, Abruzzo LV, et al. A multidrug resistance transporter from human MCF-7 breast cancer cells. Proc Natl Acad Sci USA 1998; 95:15665-15670.

6 Scherf U, Ross DT, Waltham M, et al. A gene expression database for the molecular pharmacology of cancer. Nat Genet 2000; 24:236-244.

7 Ross DT, Scherf U, Eisen MB, et al. Systematic variation in gene expression patterns in human cancer cell lines. Nat Genet 2000; 24:227-235.

8 Staunton JE, Slonim DK, Coller HA, Tamayo P, Angelo MJ, Park $\mathrm{J}$, et al. Chemosensitivity prediction by transcriptional profiling. Proc Natl Acad Sci USA 2001; 98:10787-10792.

9 Dan S, Tsunoda T, Kitahara O, et al. An integrated database of chemosensitivity to 55 anticancer drugs and gene expression profiles of 39 human cancer cell lines. Cancer Res 2002; 62:11391147.

10 Yasui K, Mihara S, Zhao C, et al. Alteration in copy numbers of genes as a mechanism for acquired drug resistance. Cancer Res 2004; 64:1403-1410.

11 Krishnamurthy PC, Du G, Fukuda Y, et al. Identification of a mammalian mitochondrial porphyrin transporter. Nature 2006; 443:586-589.

12 Gyorffy B, Surowiak P, Kiesslich O, et al. Gene expression profiling of 30 cancer cell lines predicts resistance towards 11 anticancer drugs at clinically achieved concentrations. Int $\mathrm{J}$ Cancer 2006; 118:1699-1712.
13 Huang Y, Anderle P, Bussey KJ, et al. Membrane transporters and channels: role of the transportome in cancer chemosensitivity and chemoresistance. Cancer Res 2004; 64:4294-4301.

14 Chen H, Liu J, Merrick BA, Waalkes MP. Genetic events associated with arsenic-induced malignant transformation: applications of cDNA microarray technology. Mol Carcinog 2001; 30:7987.

15 Liu J, Chen H, Miller DS, et al. Overexpression of glutathione S-transferase II and multidrug resistance transport proteins is associated with acquired tolerance to inorganic arsenic. Mol Pharmacol 2001; 60:302-309.

16 Voigt W, Vanhoefer U, Yin MB, Minderman H, Schmoll HJ, Rustum YM. Evaluation of topoisomerase I catalytic activity as determinant of drug response in human cancer cell lines. Anticancer Res 1997; 17:3707-3711.

17 Komatani H, Kotani H, Hara Y, Nakagawa R, Matsumoto M, Arakawa $\mathrm{H}$, et al. Identification of breast cancer resistant protein/mitoxantrone resistance/placenta-specific, ATP-binding cassette transporter as a transporter of NB-506 and J-107088, topoisomerase I inhibitors with an indolocarbazole structure. Cancer Res 2001; 61:2827-2832.

18 Tan Y, Li G, Zhao C, et al. Expression of sorcin predicts poor outcome in acute myeloid leukemia. Leuk Res 2003; 27:125131.

19 Lamendola DE, Duan Z, Yusuf RZ, Seiden MV. Molecular description of evolving paclitaxel resistance in the SKOV-3 human ovarian carcinoma cell line. Cancer Res 2003; 63:2200-2205.

20 Goto T, Takano M, Sakamoto M, et al. Gene expression profiles with cDNA microarray reveal RhoGDI as a predictive marker for paclitaxel resistance in ovarian cancers. Oncol Rep 2006; 15:1265-1271.

21 Xiao JJ, Huang Y, Dai Z, et al. Chemoresistance to depsipeptide FK228 [(E)-(1S,4S,10S,21R)-7-[(Z)-ethylidene]-4,21-diisopropyl-2-oxa-12,13-dithia-5,8,20,23-tetraazabicyclo[8,7,6]-tricos16-ene-3,6,9,22-pentanone] is mediated by reversible MDR1 induction in human cancer cell lines. J Pharmacol Exp Ther 2005; 314:467-475.

22 Manara MC, Perdichizzi S, Serra M, et al. The molecular mechanisms responsible for resistance to ET-743 (Trabectidin; Yondelis) in the Ewing's sarcoma cell line, TC-71. Int J Oncol 2005; 27:1605-1616.

23 Pang E, Hu Y, Chan KY, et al. Karyotypic imbalances and differential gene expressions in the acquired doxorubicin resistance of hepatocellular carcinoma cells. Lab Invest 2005; 85:664-674.

24 Gillet JP, Efferth T, Steinbach D, et al. Microarray-based detection of multidrug resistance in human tumor cells by expression profiling of ATP-binding cassette transporter genes. Cancer Res 2004; 64:8987-8993.

25 Park S, Shimizu C, Shimoyama T, et al. Gene expression profiling of ATP-binding cassette (ABC) transporters as a predictor of the pathologic response to neoadjuvant chemotherapy in breast cancer patients. Breast Cancer Res Treat 2006; 99:9-17.

26 Pratt S, Shepard RL, Kandasamy RA, Johnston PA, Perry W 3rd, Dantzig AH. The multidrug resistance protein 5 (ABCC5) confers resistance to 5-fluorouracil and transports its monophosphorylated metabolites. Mol Cancer Ther 2005; 4:855-863.

27 Wielinga P, Hooijberg JH, Gunnarsdottir S, et al. The human multidrug resistance protein MRP5 transports folates and can mediate cellular resistance against antifolates. Cancer Res 2005; 
65:4425-4430.

28 Gillet JP, Schneider J, Bertholet V, De Longueville F, Remacle J, Efferth T. Microarray expression profiling of ABC transporters in human breast cancer. Cancer Genomics Proteomics 2006; 3:97-106.

29 Mutch DM, Anderle P, Fiaux M, et al. Regional variations in $\mathrm{ABC}$ transporter expression along the mouse intestinal tract. Physiol Genomics 2004; 17:11-20.

30 Annereau JP, Szakacs G, Tucker CJ, et al. Analysis of ATPBinding Cassette Transporter Expression in Drug-Selected Cell Lines by a Microarray Dedicated to Multidrug Resistance. Mol Pharmacol 2004; 66:1397-1405.

31 Kimmig A, Gekeler V, Neumann M, et al. Susceptibility of multidrug-resistant human leukemia cell lines to human interleukin 2-activated killer cells. Cancer Res 1990; 50:6793-6799.

32 Brugger D, Herbart H, Gekeler V, et al. Functional analysis of P-glycoprotein and multidrug resistance associated protein related multidrug resistance in AML-blasts. Leuk Res 1999; 23:467-475.

33 Steinbach D, Gillet JP, Sauerbrey A, et al. ABCA3 as a possible cause of drug resistance in childhood acute myeloid leukemia. Clin Cancer Res 2006; 12:4357-4363.

34 Yamano G, Funahashi H, Kawanami O, et al. ABCA3 is a lamellar body membrane protein in human lung alveolar type II cells. FEBS Lett 2001; 508:221-225.

35 Efferth T, Gillet JP, Sauerbrey A, et al. Expression profiling of ATP-binding cassette transporters in childhood T-cell acute lymphoblastic leukemia. Mol Cancer Ther 2006; 5:1986-1994.

36 Szakacs G, Annereau JP, Lababidi S, et al. Predicting drug sensitivity and resistance: profiling $\mathrm{ABC}$ transporter genes in cancer cells. Cancer Cell 2004; 6:129-137.

37 Ludwig JA, Szakacs G, Martin SE, et al. Selective toxicity of NSC73306 in MDR1-positive cells as a new strategy to circumvent multidrug resistance in cancer. Cancer Res 2006; 66:48084815 .

38 Langmann T, Mauerer R, Zahn A, et al. Real-time reverse transcription-PCR expression profiling of the complete human ATP-binding cassette transporter superfamily in various tissues. Clin Chem 2003; 49:230-238.

39 Langmann T, Mauerer R, Schmitz G. Human ATP-binding cassette transporter TaqMan low-density array: analysis of macrophage differentiation and foam cell formation. Clin Chem 2006; 52:310-313.

40 Liu Y, Peng H, Zhang JT. Expression Profiling of ABC Transporters in a Drug-Resistant Breast Cancer Cell Line Using AmpArray. Mol Pharmacol 2005; 68:430-438.

41 Kallioniemi A, Kallioniemi OP, Sudar D, et al. Comparative genomic hybridization for molecular cytogenetic analysis of solid tumors. Science 1992; 258:818-821.
42 Boonstra R, Timmer-Bosscha H, van Echten-Arends J, et al. Mitoxantrone resistance in a small cell lung cancer cell line is associated with ABCA2 upregulation. Br J Cancer 2004; 90:2411-2417.

43 Wilson MW, Fraga CH, Fuller CE, et al. Immunohistochemical detection of multidrug-resistant protein expression in retinoblastoma treated by primary enucleation. Invest Ophthalmol Vis Sci 2006; 47:1269-1273.

44 Zhang W, Shannon WD, Duncan J, Scheffer GL, Scheper RJ, McLeod HL. Expression of drug pathway proteins is independent of tumour type. J Pathol 2006; 209:213-219.

45 Lee JK, Bussey KJ, Gwadry FG, et al. Comparing cDNA and oligonucleotide array data: concordance of gene expression across platforms for the NCI-60 cancer cells. Genome Biol 2003; 4:R82.

46 Brown KJ, Fenselau C. Investigation of doxorubicin resistance in MCF-7 breast cancer cells using shot-gun comparative proteomics with proteolytic $18 \mathrm{O}$ labeling. J Proteome Res 2004; 3:455-462.

47 Gehrmann ML, Hathout Y, Fenselau C. Evaluation of metabolic labeling for comparative proteomics in breast cancer cells. J Proteome Res 2004; 3:1063-1068.

48 Isidoro A, Casado E, Redondo A, et al. Breast carcinomas fulfill the Warburg hypothesis and provide metabolic markers of cancer prognosis. Carcinogenesis 2005; 26:2095-2104.

49 Calcagno AM, Chewning KJ, Wu CP, Ambudkar SV. Plasma membrane calcium ATPase (PMCA4): a housekeeper for RTPCR relative quantification of polytopic membrane proteins. BMC Mol Biol 2006; 7:29.

50 Han B, Zhang JT. Multidrug resistance in cancer chemotherapy and xenobiotic protection mediated by the half ATP-binding cassette transporter ABCG2. Curr Med Chem Anti-Canc Agents 2004; 4:31-42.

51 Zhang JT, Ling V. Study of membrane orientation and glycosylated extracellular loops of mouse P-glycoprotein by in vitro translation. J Biol Chem 1991; 266:18224-18232.

52 Zhang JT, Duthie M, Ling V. Membrane topology of the N-terminal half of the hamster P-glycoprotein molecule. J Biol Chem 1993; 268:15101-15110.

53 Skach WR, Calayag MC, Lingappa VR. Evidence for an alternate model of human P-glycoprotein structure and biogenesis. J Biol Chem 1993; 268:6903-6908.

54 Chen Q, Yang Y, Liu Y, Han B, Zhang JT. Cytoplasmic retraction of the amino terminus of human multidrug resistance protein 1. Biochemistry 2002; 41:9052-9062.

55 Chen Q, Yang Y, Li L, Zhang JT. The Amino Terminus of the Human Multidrug Resistance Transporter ABCC1 Has a U-shaped Folding with a Gating Function. J Biol Chem 2006; 281:3115231163. 